\title{
On the number of subsequences with a given sum in a finite abelian group
}

\author{
Gerard Jennhwa Chang, ${ }^{123 *}$ Sheng-Hua Chen, ${ }^{13 \dagger}$ \\ Yongke Qu, ${ }^{4 \ddagger}$ Guoqing Wang, ${ }^{5 \S}$ and Haiyan Zhang ${ }^{6 \nsubseteq}$ \\ ${ }^{1}$ Department of Mathematics, National Taiwan University, Taipei 10617, Taiwan \\ ${ }^{2}$ Taida Institute for Mathematical Sciences, National Taiwan University, Taipei 10617, Taiwan \\ ${ }^{3}$ National Center for Theoretical Sciences, Taipei Office \\ ${ }^{4}$ Center for Combinatorics, LPMC-TJKLC, Nankai University, Tianjin 300071, P.R. China \\ ${ }^{5}$ Department of Mathematics, Tianjin Polytechnic University, Tianjin 300160, P.R. China \\ ${ }^{6}$ Department of Mathematics, Harbin University of Science and Technology, Harbin 150080, P.R. China
}

Submitted: Jan 24, 2011; Accepted: June 10, 2011; Published: Jun 21, 2011

Mathematics Subject Classifications: 11B75, 11R27, 20K01

\begin{abstract}
Suppose $G$ is a finite abelian group and $S$ is a sequence of elements in $G$. For any element $g$ of $G$, let $N_{g}(S)$ denote the number of subsequences of $S$ with sum g. The purpose of this paper is to investigate the lower bound for $N_{g}(S)$. In particular, we prove that either $N_{g}(S)=0$ or $N_{g}(S) \geq 2^{|S|-D(G)+1}$, where $D(G)$ is the smallest positive integer $\ell$ such that every sequence over $G$ of length at least $\ell$ has a nonempty zero-sum subsequence. We also characterize the structures of the extremal sequences for which the equality holds for some groups.
\end{abstract}

\section{Introduction}

Suppose $G$ is a finite abelian group and $S$ is a sequence over $G$. The enumeration of subsequences with certain prescribed properties is a classical topic in Combinatorial Number

\footnotetext{
*E-mail: gjchang@math.ntu.edu.tw. Supported in part by the National Science Council under grant NSC98-2115-M-002-013-MY3.

†E-mail: b91201040@ntu.edu.tw.

†E-mail: quyongke@sohu.com.

$\S$ E-mail: gqwang1979@yahoo.com.cn. Supported by NSFC (11001035).

『E-mail: yanhaizhang2222@sohu.com.
} 
Theory going back to Erdös, Ginzburg and Ziv $[6,14,15]$ who proved that $2 n-1$ is the smallest integer such that every sequence $S$ over a cyclic group $C_{n}$ has a subsequence of length $n$ with zero-sum. This raises the problem of determining the smallest positive integer $\ell$ such that every sequence $S$ of length at least $\ell$ has a nonempty zero-sum subsequence. Such an integer $\ell$ is called the Davenport constant [4] of $G$, denoted by $D(G)$, which is still unknown in general.

For any $g$ of $G$, let $N_{g}(S)$ denote the number of subsequences of $S$ with sum $g$. In 1969, J. E. Olson [24] proved that $N_{0}(S) \geq 2^{|S|-D(G)+1}$ for every sequence $S$ over $G$ of length $|S| \geq D(G)$. Subsequently, several authors $[1,2,3,5,8,9,11,13,16,17,18,20]$ obtained a huge variety of results on the number of subsequences with prescribed properties. However, for any arbitrary $g$ of $G$, the lower bound of $N_{g}(S)$ remains undetermined.

In this paper, we determine the best possible lower bound of $N_{g}(S)$ for an arbitrary $g$ of $G$. We also characterize the structures of the extremal sequences which attain the lower bound for some groups.

\section{Notation and lower bound}

Our notation and terminology are consistent with [10]. We briefly gather some notions and fix the notation concerning sequences over abelian group. Let $\mathbb{N}$ and $\mathbb{N}_{0}$ denote the sets of positive integers and non-negative integers, respectively. For integers $a, b \in \mathbb{N}_{0}$, we set $[a, b]=\left\{x \in \mathbb{N}_{0}: a \leq x \leq b\right\}$. Throughout, all abelian groups are written additively. For a positive integer $n$, let $C_{n}$ denote a cyclic group with $n$ elements.

For a sequence $S=g_{1} \cdot \ldots \cdot g_{m}$ of elements in $G$, we use $\sigma(S)=\sum_{i=1}^{m} g_{i}$ denote the sum of $S$. By $\lambda$ we denote the empty sequence and adopt the convention that $\sigma(\lambda)=0$. A subsequence $T \mid S$ means $T=g_{i_{1}} \cdot \ldots \cdot g_{i_{k}}$ with $\left\{i_{1}, \ldots, i_{k}\right\} \subseteq[1, m]$; we denote by $I_{T}$ the index set $\left\{i_{1}, \ldots, i_{k}\right\}$ of $T$, and identify two subsequences $S_{1}$ and $S_{2}$ if $I_{S_{1}}=I_{S_{2}}$. We denote $-T=\left(-g_{i_{1}}\right) \cdot \ldots \cdot\left(-g_{i_{k}}\right)$. Let $S_{1}, \ldots, S_{n}$ be $n$ subsequences of $S$, denote by $\operatorname{gcd}\left(S_{1}, \ldots, S_{n}\right)$ the subsequence of $S$ with index set $I_{S_{1}} \bigcap \cdots \bigcap I_{S_{n}}$. We say two subsequences $S_{1}$ and $S_{2}$ are disjoint if $\operatorname{gcd}\left(S_{1}, S_{2}\right)=\lambda$. If $S_{1}$ and $S_{2}$ are disjoint, then we denote by $S_{1} S_{2}$ the subsequence with index set $I_{S_{1}} \cup I_{S_{2}}$; if $S_{1} \mid S_{2}$, we denote by $S_{2} S_{1}^{-1}$ the subsequence with index set $I_{S_{2}} \backslash I_{S_{1}}$. Define $\sum(S)=\left\{\sum_{i \in I} g_{i}: \phi \neq I \subseteq[1, m]\right\}$, and $\sum \bullet(S)=\sum(S) \cup\{0\}$.

The sequence $S$ is called

- a zero-sum sequence if $\sigma(S)=0$,

- a zero-sum free sequence if $0 \notin \sum(S)$,

- a minimal zero-sum sequence if $S \neq \lambda, \sigma(S)=0$, and every $T \mid S$ with $1 \leq|T|<|S|$ is zero-sum free,

- a unique factorial sequence if $0 \nmid S$ and if $S=T_{1} \cdot \ldots \cdot T_{k} S^{\prime}$, where $T_{1}, \ldots, T_{k}$ are all the minimal zero-sum subsequences of $S$.

Define

$$
\mathscr{N}_{1}(G)=\max \{|S|: S \text { is a unique factorial sequence over } G\}
$$


where the maximum is taken when $S$ runs over all unique factorial sequences over $G$.

Remark 1. The concept of unique factorial sequence was first introduced by Narkiewicz in [21] for zero-sum sequence. For recent progress on unique factorial sequences we refer to $[12]$.

For an element $g$ of $G$, let

$$
N_{g}(S)=\mid\left\{I_{T}: T \mid S \text { and } \sigma(T)=g\right\} \mid
$$

denote the number of subsequences $T$ of $S$ with sum $\sigma(T)=g$. Notice that we always have $N_{0}(S) \geq 1$.

Theorem 2. If $S$ is a sequence over a finite abelian group $G$ and $g \in \sum^{\bullet}(S)$, then $N_{g}(S) \geq 2^{|S|-D(G)+1}$.

Proof. We shall prove the theorem by induction on $m=|S|$. The case of $m \leq D(G)-$ 1 is clear. We now consider the case of $m \geq D(G)$. Choose a subsequence $T \mid S$ of minimum length with $\sigma(T)=g$, and a nonempty zero-sum subsequence $W \mid T\left(-\left(S T^{-1}\right)\right.$ ). By the minimality of $|T|, W$ is not a subsequence of $T$, for otherwise $T W^{-1}$ is a shorter subsequence of $S$ with $\sigma\left(T W^{-1}\right)=g$. Choose a term $a \mid W$ with $a \nmid T$, and let $X=$ $\operatorname{gcd}(W, T)$. Then, $-a \mid S T^{-1}$ such that $g=\sigma(T) \in \sum^{\bullet}\left(S(-a)^{-1}\right)$ and $(g-\sigma(X))-$ $(0-\sigma(X)-a)=g+a=\sigma\left(T X^{-1}\left(-\left(W(X a)^{-1}\right)\right)\right) \in \sum^{\bullet}\left(S(-a)^{-1}\right)$. By the induction hypothesis, $N_{g}(S)=N_{g}\left(S(-a)^{-1}\right)+N_{g+a}\left(S(-a)^{-1}\right) \geq 2^{m-D(G)}+2^{m-D(G)}=2^{m-D(G)+1}$. This completes the proof of the theorem.

Notice that the result in $[24]$ that $N_{0}(S) \geq 2^{|S|-D(G)+1}$ for any sequence $S$ over $G$, together with the following lemma, also gives Theorem 2.

Lemma 3. If $S$ is a sequence over a finite abelian group $G$, then for any $T \mid S$ with $\sigma(T)=g \in \sum^{\bullet}(S)$,

$$
N_{g}(S)=N_{0}\left(T\left(-\left(S T^{-1}\right)\right)\right) .
$$

Proof. Let $\mathcal{A}=\{X \mid S: \sigma(X)=g\}$ and $\mathcal{B}=\left\{Y \mid T\left(-\left(S T^{-1}\right)\right): \sigma(Y)=0\right\}$. It is clear that $|\mathcal{A}|=N_{g}(S)$ and $|\mathcal{B}|=N_{0}\left(T\left(-\left(S T^{-1}\right)\right)\right)$. Define the map $\varphi: \mathcal{A} \rightarrow \mathcal{B}$ by $\varphi(X)=T X_{1}^{-1}\left(-X_{2}\right)$ for any $X \in \mathcal{A}$, where $X_{1}=\operatorname{gcd}(X, T)$ and $X_{2}=\operatorname{gcd}\left(X, S T^{-1}\right)$. It is straightforward to check that $\varphi$ is a bijection, which implies $N_{g}(S)=N_{0}\left(T\left(-\left(S T^{-1}\right)\right)\right.$ ).

We remark that the lower bound in Theorem 2 is best possible. For any $g \in G$ and any $m \geq D(G)-1$, we construct the extremal sequence $S$ over $G$ of length $m$ with respect to $g$ as follows: Take a zero-sum free sequence $U$ over $G$ with $|U|=D(G)-1$. Clearly, $U$ contains a subsequence $T$ with $\sigma(T)=g$. For $S=T\left(-\left(U T^{-1}\right)\right) 0^{m-D(G)+1}$, by Lemma 3, $N_{g}(S)=N_{0}\left(U 0^{m-D(G)+1}\right)=2^{m-D(G)+1}$.

Proposition 4. If $S$ is a sequence over a finite abelian group $G$ such that $N_{h}(S)=$ $2^{|S|-D(G)+1}$ for some $h \in G$, then $N_{g}(S) \geq 2^{|S|-D(G)+1}$ for all $g \in G$.

Proof. If there exists $g$ such that $N_{g}(S)<2^{|S|-D(G)+1}$, then

$$
N_{h}(S(h-g))=N_{h}(S)+N_{g}(S)<2^{|S|+1-D(G)+1}
$$

is a contradiction to Theorem 2 since $h \in \sum^{\bullet}(S) \subseteq \sum^{\bullet}(S(h-g))$. 


\section{The structures of extremal sequences}

In this section, we study sequence $S$ for which $N_{g}(S)=2^{|S|-D(G)+1}$. By Lemma 3, we need only pay attention to the case $g=0$. Also, as $N_{g}(0 S)=2 N_{g}(S)$, it suffices to consider the case $0 \nmid S$. For $|S| \geq D(G)-1$, define

$$
E(S)=\left\{g \in G: N_{g}(S)=2^{|S|-D(G)+1}\right\} .
$$

Lemma 5. Suppose $S$ is a sequence over a finite abelian group $G$ with $0 \nmid S,|S| \geq D(G)$ and $0 \in E(S)$. If $a$ is a term of a zero-sum subsequence $T$ of $S$, then

$$
E(S)+\{0,-a\} \subseteq E\left(S a^{-1}\right) .
$$

Proof. Since $0,-a \in \sum^{\bullet}\left(S a^{-1}\right)$, by Theorem $2, N_{0}\left(S a^{-1}\right) \geq 2^{|S|-D(G)}$ and $N_{-a}\left(S a^{-1}\right) \geq$ $2^{|S|-D(G)}$. On the other hand, $N_{0}\left(S a^{-1}\right)+N_{-a}\left(S a^{-1}\right)=N_{0}(S)=2^{|S|-D(G)+1}$ and so $N_{0}\left(S a^{-1}\right)=N_{-a}\left(S a^{-1}\right)=2^{|S|-D(G)}$. Hence, by Proposition $4, N_{g}\left(S a^{-1}\right) \geq 2^{|S|-D(G)}$ for all $g \in G$. Now, for every $h \in E(S), N_{h}\left(S a^{-1}\right)+N_{h-a}\left(S a^{-1}\right)=N_{h}(S)=2^{|S|-D(G)+1}$ and so $N_{h}\left(S a^{-1}\right)=N_{h-a}\left(S a^{-1}\right)=2^{|S|-D(G)}$, i.e., $\{h, h-a\} \subseteq E\left(S a^{-1}\right)$. This proves $E(S)+\{0,-a\} \subseteq E\left(S a^{-1}\right)$

Lemma 6 ([14], Lemma 6.1.3, Lemma 6.1.4). Let $G \cong C_{n_{1}} \oplus C_{n_{2}} \oplus \cdots \oplus C_{n_{r}}$ with $n_{1}\left|n_{2}\right| \cdots \mid n_{r}$, and $H$ be a subgroup of $G$, then $D(G) \geq D(H)+D(G / H)-1$ and $D(G) \geq$ $\sum_{i=1}^{r}\left(n_{i}-1\right)+1$.

Lemma 7. If $S$ is a sequence over a finite abelian group $G$ such that $E(S)$ contains a non-trivial subgroup $H$ of $G$, then $H \cong \bigoplus_{i=1}^{r} C_{2}$ and $D(G)=D(G / H)+r$.

Proof. Suppose $H \cong C_{n_{1}} \oplus C_{n_{2}} \oplus \cdots \oplus C_{n_{r}}$, where $n_{1}\left|n_{2}\right| \cdots \mid n_{r}$, and assume that $S=g_{1} \cdot \ldots \cdot g_{m}$. Consider the canonical map $\varphi: G \rightarrow G / H$ and let $\varphi(S)=\varphi\left(g_{1}\right) \cdot \ldots \cdot \varphi\left(g_{m}\right)$ be a sequence over $G / H$. Then

$$
|H| \cdot 2^{|S|-D(G)+1}=\sum_{h \in H} N_{h}(S)=N_{0}(\varphi(S)) \geq 2^{|\varphi(S)|-D(G / H)+1} .
$$

It follows from Lemma 6 that $|H| \geq 2^{D(G)-D(G / H)} \geq 2^{D(H)-1}$, and so

$$
\prod_{i=1}^{r} n_{i} \geq 2^{\sum_{i=1}^{r}\left(n_{i}-1\right)}=\prod_{i=1}^{r} 2^{n_{i}-1} .
$$

Hence, $n_{i}=2$ for all $i$, which gives $H \cong \bigoplus_{i=1}^{r} C_{2}$ and $D(G)=D(G / H)+r$.

Lemma 8. ([22], Proposition 9; [12], Lemma 3.9) Let $G$ be a finite abelian group, and let $S=S_{1} \cdot \ldots \cdot S_{r}$ be a unique factorial zero-sum sequence over $G$, where $S_{1}, \ldots, S_{r}$ are all the minimal zero-sum subsequences of $S$. Then, $\left|S_{1}\right| \cdots\left|S_{r}\right| \leq|G|$.

Lemma 9. Let $G$ be a finite abelian group, and let $S=S_{1} \cdot \ldots \cdot S_{r} S^{\prime}$ be a unique factorial sequence over $G$, where $S_{1}, \ldots, S_{r}$ are all the minimal zero-sum subsequences of $S$ and $S^{\prime}$ is empty or zero-sum free. Then, $\left|S_{1}\right| \cdots\left|S_{r}\right| \max \left\{1,\left|S^{\prime}\right|\right\} \leq|G|$. 
Proof. If $\left|S^{\prime}\right| \leq 1$ then $\left|S_{1}\right| \cdots\left|S_{r}\right| \max \left\{1,\left|S^{\prime}\right|\right\}=\left|S_{1}\right| \cdots\left|S_{r}\right| \leq|G|$ follows from Lemma 8. Now assume that $\left|S^{\prime}\right| \geq 2$. In a similar way to the proof of Proposition 9 in [22] (or Lemma 3.9 in [12]) one can prove that $\left|S_{1}\right| \cdots\left|S_{r}\right|\left|S^{\prime}\right| \leq|G|$.

Lemma 10. If $G$ is a finite abelian group then $\mathscr{N}_{1}(G) \leq \log _{2}|G|+D(G)-1$.

Proof. Let $S$ be a unique factorial sequence over $G$ with $|S|=\mathscr{N}_{1}(G)$. Then, $S=$ $S_{1} \cdot \ldots \cdot S_{r} S^{\prime}$ with $S_{1}, \ldots, S_{r}$ are all the minimal zero-sum subsequences of $S$. By Lemma 9, $\left|S_{1}\right| \cdots\left|S_{r}\right| \leq|G|$. It follows from $\left|S_{i}\right| \geq 2$ for every $i \in[1, r]$ that $r \leq \log _{2}|G|$. Take an element $x_{i} \in S_{i}$ for every $i \in[1, r]$. Since $S_{1}, \ldots, S_{r}$ are all the minimal zero-sum subsequences of $S$, we have that $S_{1} \cdot \ldots \cdot S_{r} S^{\prime}\left(x_{1} \cdot \ldots \cdot x_{r}\right)^{-1}$ is zero-sum free. It follows that $|S|-r=\left|S_{1} \cdot \ldots \cdot S_{r} S^{\prime}\right|-r \leq D(G)-1$. Therefore, $\mathscr{N}_{1}(G)=|S| \leq \log _{2}|G|+D(G)-1$.

Now, we consider the case $G=C_{n}$. Notice that $D\left(C_{n}\right)=n$.

Theorem 11. For $n \geq 3$, if $S$ is a sequence over the cyclic group $C_{n}$ with $0 \nmid S$ and $N_{0}(S)=2^{|S|-n+1}$, then $n-1 \leq|S| \leq n$ and $S=a^{|S|}$, where $a$ generates $C_{n}$.

Proof. Suppose $S$ is a sequence over the cyclic group $C_{n}$ with $0 \nmid S$ and $N_{0}(S)=2^{|S|-n+1}$. We first show by induction that

$$
S=a^{|S|}
$$

where $\langle a\rangle=C_{n}$. For $|S|=n-1$, we have $N_{0}(S)=1$, i.e., $S$ is a zero-sum free sequence, and (1) follows readily.

For $|S| \geq n$, since $N_{0}(S)=2^{|S|-n+1} \geq 2, S$ contains at least one nonempty zerosum subsequence $T$. Take an arbitrary term $c$ from $T$. By $\operatorname{Lemma} 5,0 \in E\left(S c^{-1}\right)$. It follows from the induction hypothesis that $S c^{-1}=a^{|S|-1}$ for some a generating $C_{n}$. By the arbitrariness of $c$, we conclude that (1) holds.

To prove $|S| \leq n$, we suppose to the contrary that $|S| \geq n+1$. By (1) and Lemma 5 ,

$$
0 \in E\left(a^{n+1}\right) .
$$

We see that $N_{0}\left(a^{n+1}\right) \geq 1+\left(\begin{array}{c}n+1 \\ n\end{array}\right)>4$, a contraction with (2).

Notice that Theorem 11 is not true for $n=2$, since for any sequence $S$ over $C_{2}$ with $0 \nmid S$, we always have $N_{0}(S)=2^{|S|-2+1}$.

While the structure of a sequence $S$ over a general finite abelian group $G$ with $0 \nmid S$ and $N_{0}(S)=2^{|S|-D(G)+1}$ is still not known, we have the following result for the case when $|G|$ is odd.

Theorem 12. If $S$ is a sequence over a finite abelian group $G$ of odd order with $0 \nmid S$ and $N_{0}(S)=2^{|S|-D(G)+1}$, then $S$ is unique factorial and the number of minimal zero-sum subsequences of $S$ is $|S|-D(G)+1$, and therefore $|S| \leq \mathscr{N}_{1}(G) \leq D(G)-1+\log _{2}|G|$.

Proof. We first note that if $S$ is a unique factorial sequence, i.e., $S=S_{1} \cdot \ldots \cdot S_{\ell} S^{\prime}$ where $S_{1}, \ldots, S_{\ell}$ are all the minimal zero-sum subsequences of $S$, then $2^{\ell}=N_{0}(S)=2^{|S|-D(G)+1}$, which implies that $\ell=|S|-D(G)+1$, and that $|S| \leq \mathscr{N}_{1}(G) \leq \log _{2}|G|+D(G)-1$ follows from Lemma 10. Therefore, it suffices to show that $S$ is a unique factorial sequence. 
We proceed by induction on $|S|$. If $|S|=D(G)$, then $N_{0}(S)=2$ and so $S$ contains exactly one nonempty zero-sum subsequence, and we are done. Now assume

$$
|S| \geq D(G)+1
$$

If all the minimal zero-sum subsequences of $S$ are pairwise disjoint, then the conclusion follows readily. So we may assume that there exist two distinct minimal zero-sum subsequences $T_{1}$ and $T_{2}$ with $\operatorname{gcd}\left(T_{1}, T_{2}\right) \neq \lambda$. Take a term $a \mid \operatorname{gcd}\left(T_{1}, T_{2}\right)$. By Lemma 5, $0 \in E\left(S a^{-1}\right)$ and so $S a^{-1}$ contains $r=|S|-D(G) \geq 1$ pairwise disjoint minimal zerosum subsequences $T_{3}, T_{4}, \ldots, T_{r+2}$ by the induction hypothesis. Now we need the following claim.

Claim A. There is no term which is contained in exactly one $T_{i}$, where $i \in[1, r+2]$.

Proof of Claim A. Assume to the contrary that, there is a term $b$ such that $b \mid T_{t}$ for some $t \in[1, r+2]$, and such that $b \nmid T_{i}$ for every $i \in[1, r+2] \backslash\{t\}$. By Lemma 5, we have $0 \in E\left(S b^{-1}\right)$. It follows from the induction hypothesis that $S b^{-1}$ contains exactly $r$ minimal zero-sum subsequences, which is a contradiction. This proves Claim A.

Choose a term $c$ in $T_{1}$ but not in $T_{2}$. By Claim $\mathrm{A}$, we have that $c$ is in another $T_{i}$, say $T_{r+2}$ and so not in any of $T_{3}, T_{4}, \ldots, T_{r+1}$. Again $S c^{-1}$ contains exactly $r$ disjoint minimal zero-sum subsequences, which are just $T_{2}, T_{3}, \ldots, T_{r+1}$. If $r \geq 2$, noticing that $\operatorname{gcd}\left(T_{r+1}, T_{i}\right)=\lambda$ for every $i \in[2, r+2] \backslash\{r+1\}$, it follows from Claim A that $T_{r+1} \mid T_{1}$, which is a contradiction to the minimality of $T_{1}$. Therefore,

$$
r=1 \text {. }
$$

Then $N_{0}(S)=4$ and $T_{1}, T_{2}, T_{3}$ are all the minimal zero-sum subsequences of $S$. If there is some $d \mid \operatorname{gcd}\left(T_{1}, T_{2}, T_{3}\right)$, then $S d^{-1}$ contains no minimal zero-sum subsequence, which is impossible. Thus $\operatorname{gcd}\left(T_{1}, T_{2}, T_{3}\right)=\lambda$. Let $X=\operatorname{gcd}\left(T_{2}, T_{3}\right), Y=\operatorname{gcd}\left(T_{1}, T_{3}\right)$ and $Z=\operatorname{gcd}\left(T_{1}, T_{2}\right)$. It follows from Claim A that $T_{1}=Y Z, T_{2}=X Z$ and $T_{3}=X Y$. Therefore, $\sigma(Y)+\sigma(Z)=\sigma(X)+\sigma(Z)=\sigma(X)+\sigma(Y)=0$. This gives that $2 \sigma(X)=$ $2 \sigma(Y)=2 \sigma(Z)=0$. Since $|G|$ is odd, it follows that $\sigma(X)=0$, which is a contradiction. This completes the proof of the theorem.

If we further assume that $E(S)=\{0\}$ in Theorem 12, the structure of $S$ can be further restricted.

Corollary 13. If $S$ is a sequence over a finite abelian group $G$ of odd order with $0 \nmid S$ and $E(S)=\{0\}$, then $S$ is a unique factorial zero-sum sequence and the number of minimal zero-sum subsequences of $S$ is $|S|-D(G)+1$. Therefore, $|S| \leq \mathscr{N}_{1}(G) \leq$ $\log _{2}|G|+D(G)-1$.

Proof. By Theorem 12, $S$ is unique factorial and contains exactly $r=|S|-D(G)+1$ minimal zero-sum subsequences $T_{1}, \ldots, T_{r}$ (say). Therefore, $S=T_{1} \cdot \ldots \cdot T_{r} W$. For any subsequence $X$ of $S$ with $\sigma(X)=\sigma(W)$, if $W \nmid X$, then $S X^{-1}$ is a zero-sum subsequence containing terms in $W$, which is impossible. So $W \mid X$, and then $\sigma\left(X W^{-1}\right)=0$. This gives $X=T_{i_{1}} \cdot \ldots \cdot T_{i_{s}} W$ with $1 \leq i_{1}<\cdots<i_{s} \leq r$. Hence, $N_{\sigma(W)}(S)=2^{r}$ and then $\sigma(W) \in E(S)=\{0\}$ implying $W=\lambda$. Now $|S| \leq \mathscr{N}_{1}(G) \leq \log _{2}|G|+D(G)-1$ follows from Lemma 10. 
Remark 14. The following example shows that Theorem 12 does not hold for all finite abelian groups. Let $G=C_{2} \oplus C_{2 n_{1}} \oplus \cdots \oplus C_{2 n_{r}}=\langle e\rangle \oplus\left\langle e_{1}\right\rangle \oplus \cdots \oplus\left\langle e_{r}\right\rangle$ with $1 \leq n_{1}|\cdots| n_{r}$ and $D(G)=d^{*}(G)+1$. For any $m \geq D(G)+1$, take $S=e^{m-D(G)+2} \cdot \prod_{i=1}^{r} e_{i}^{2 n_{i}-1}$. It is easy to check that $N_{0}(S)=\left(\begin{array}{l}k \\ 0\end{array}\right)+\left(\begin{array}{l}k \\ 2\end{array}\right)+\cdots+\left(\begin{array}{c}k \\ 2\left\lfloor\frac{k}{2}\right\rfloor\end{array}\right)=2^{k-1}$ where $k=m-D(G)+2$, and that $S$ is not a unique factorial sequence.

The property that $S$ contains exactly $|S|-D(G)+1$ minimal zero-sum subsequences, all of which are pairwise disjoint, implies that $|S|$ is bounded as in the case of Theorem 11 for cyclic groups. In general, we have the following theorem.

Theorem 15. For any finite abelian group $G \cong C_{n_{1}} \oplus C_{n_{2}} \oplus \cdots \oplus C_{n_{r}}$ with $n_{1}\left|n_{2}\right| \cdots \mid n_{r}$, (i) implies the three equivalent statements (ii), (iii) and (iv).

(i) Any sequence $S$ over $G$ with $0 \nmid S$ and $N_{0}(S)=2^{|S|-D(G)+1}$, contains exactly $|S|-D(G)+1$ minimal zero-sum subsequences, all of which are pairwise disjoint.

(ii) There is a natural number $t=t(G)$ such that $|S| \leq t$ for every sequence $S$ over $G$ with $0 \nmid S$ and $N_{0}(S)=2^{|S|-D(G)+1}$.

(iii) For any subgroup $H$ of $G$ isomorphic to $C_{2}, D(G) \geq D(G / H)+2$.

(iv) For any sequence $S$ over $G, E(S)$ contains no non-trivial subgroup of $G$.

Proof. (i) $\Rightarrow$ (ii). Since $S$ contains exactly $|S|-D(G)+1$ minimal zero-sum subsequences, all of which are pairwise disjoint, we have that $|S| \geq 2(|S|-D(G)+1)$ which gives $|S| \leq 2 D(G)-2$.

(ii) $\Rightarrow$ (iii). Assume to the contrary that $D(G)=D(G / H)+1$ for some subgroup $H=\{0, h\}$ of $G$. Let $\varphi: G \rightarrow G / H$ be the canonical map, and let $m=D(G / H)$. We choose a sequence $S=g_{1} \cdot \ldots \cdot g_{m}$ over $G$ such that $\varphi(S)=\varphi\left(g_{1}\right) \cdot \ldots \cdot \varphi\left(g_{m}\right)$ is a minimal zero-sum sequence over $G / H$, and $\sigma(S)=h$ in $G$. Since

$$
N_{0}(S)+N_{h}(S)=N_{0}(\varphi(S))=2=2 \cdot 2^{|S|-D(G)+1}
$$

and $N_{0}(S)$ and $N_{h}(S)$ are not zero, by theorem $2, N_{0}(S)=N_{h}(S)=2^{|S|-D(G)+1}$. Since $N_{0}\left(S h^{k}\right)=N_{0}\left(S h^{k-1}\right)+N_{h}\left(S h^{k-1}\right)=N_{h}\left(S h^{k}\right)$, by induction we have $N_{0}\left(S h^{k}\right)=$ $N_{h}\left(S h^{k}\right)=2^{\left|S h^{k}\right|-D(G)+1}$ for all $k$, a contradiction to the assumption in (ii).

(iii) $\Rightarrow$ (iv). Suppose to the contrary that there exists a sequence $S$ over $G$ such that $E(S)$ contains a non-trivial subgroup $H$ of $G$. By Lemma $7, H \cong \bigoplus_{i=1}^{s} C_{2}$ and $D(G)=D(G / H)+s$. Hence, $E(S)$ contains a subgroup $H^{\prime} \cong C_{2}$. If $D(G) \geq D\left(G / H^{\prime}\right)+2$, then by Lemma $6, D(G) \geq D\left(G / H^{\prime}\right)+2 \geq D\left(H / H^{\prime}\right)+D\left(\left(G / H^{\prime}\right) /\left(H / H^{\prime}\right)\right)+1=$ $s+1+D(G / H)>D(G)$, a contradiction.

(iv) $\Rightarrow$ (ii). For $|S| \geq D(G)$, that is, $N_{0}(S)=2^{|S|-D(G)+1}>1$, there exists a nonempty zero-sum subsequence $T_{1}$ of $S$ and a term $a_{1} \mid T_{1}$. By Lemma $5,0 \in E(S) \subseteq$ $E\left(S a_{1}^{-1}\right)$. By (iv), $\left\langle-a_{1}\right\rangle \nsubseteq E\left(S a_{1}^{-1}\right)$. Let $k$ be the minimum index such that $k\left(-a_{1}\right) \notin$ 
$E\left(S a_{1}^{-1}\right)$, that is, $\left\{0,-a_{1}, \ldots,(k-1)\left(-a_{1}\right)\right\} \subseteq E\left(S a_{1}^{-1}\right)$ but $k\left(-a_{1}\right) \notin E\left(S a_{1}^{-1}\right)$. Then, $N_{(k-1)\left(-a_{1}\right)}\left(S a_{1}^{-1}\right)=2^{\left|S a_{1}^{-1}\right|-D(G)+1}$ but $N_{k\left(-a_{1}\right)}\left(S a_{1}^{-1}\right) \neq 2^{\left|S a_{1}^{-1}\right|-D(G)+1}$. Thus,

$$
N_{(k-1)\left(-a_{1}\right)}(S)=N_{(k-1)\left(-a_{1}\right)}\left(S a_{1}^{-1}\right)+N_{k\left(-a_{1}\right)}\left(S a_{1}^{-1}\right) \neq 2^{|S|-D(G)+1}
$$

and so $(k-1)\left(-a_{1}\right) \notin E(S)$. This means

$$
E(S) \subsetneq E\left(S a_{1}^{-1}\right) .
$$

If $\left|S a_{1}^{-1}\right| \geq D(G)$, a similar argument shows that there exists a nonempty zero-sum subsequence $T_{2}$ of $S a_{1}^{-1}$ and a term $a_{2} \mid T_{2}$, thus, $E\left(S a_{1}^{-1}\right) \subsetneq E\left(S a_{1}^{-1} a_{2}^{-1}\right)$. We continue this process to get $a_{1}, a_{2}, \ldots, a_{|S|-D(G)+1}$ of $S$ such that

$$
E(S) \subsetneq E\left(S a_{1}^{-1}\right) \subsetneq \cdots \subsetneq E\left(S a_{1}^{-1} a_{2}^{-1} \cdot \ldots \cdot a_{|S|-D(G)+1}^{-1}\right) .
$$

Since $\left|E\left(S a_{1}^{-1} a_{2}^{-1} \cdot \ldots \cdot a_{|S|-D(G)+1}^{-1}\right)\right| \leq|G|$, we conclude $|S| \leq D(G)+|G|-1:=t$.

\section{Concluding remarks}

We are interested in the structure of a sequence $S$ over a finite abelian group $G$ such that $N_{0}(S)=2^{|S|-D(G)+1}$. Based on the experiences in Section 3, we have the following two conjectures.

Conjecture 16. Suppose $G$ is a finite abelian group in which $D(G) \geq D(G / H)+2$ for every subgroup $H$ of $G$ isomorphic to $C_{2}$. If $S$ is a sequence over $G$ with $0 \nmid S$ and $N_{0}(S)=2^{|S|-D(G)+1}$, then $S$ contains exactly $|S|-D(G)+1$ minimal zero-sum subsequences, all of which are pairwise disjoint.

Notice that this conjecture holds when $G$ is cyclic or $|G|$ is odd. The second conjecture concerns the length of $S$.

Conjecture 17. Suppose $G \cong C_{n_{1}} \oplus C_{n_{2}} \oplus \cdots \oplus C_{n_{r}}$ where $1<n_{1}\left|n_{2}\right| \cdots \mid n_{r}$ and $D(G)=$ $d^{*}(G)+1=\sum_{i=1}^{r}\left(n_{i}-1\right)+1$. Let $S$ be a sequence over $G$ such that $0 \nmid S$ and $E(S) \neq \emptyset$ contains no non-trivial subgroup of $G$, then $|S| \leq d^{*}(G)+r$.

The following example shows that if Conjecture 17 holds, then the upper bound $d^{*}(G)+r=\sum_{i=1}^{r} n_{i}$ is best possible. Let $G \cong C_{n_{1}} \oplus C_{n_{2}} \oplus \cdots \oplus C_{n_{r}}=\left\langle e_{1}\right\rangle \oplus\left\langle e_{2}\right\rangle \oplus \cdots \oplus\left\langle e_{r}\right\rangle$ with $1<n_{1}\left|n_{2}\right| \cdots \mid n_{r}$. Clearly, $S=\prod_{i=1}^{r} e_{i}^{n_{i}}$ is an extremal sequence with respect to 0 and of length $d^{*}(G)+r$.

Acknowledgement. The authors are grateful to the referee for helpful suggestions and comments. 


\section{References}

[1] A. Bialostocki and M. Lotspeich, Some developments of the Erdős-Ginzburg-Ziv Theorem I, Sets, Graphs and Numbers, Coll. Math. Soc. J. Bolyai 60 (1992), 97-117.

[2] E. Balandraud, An addition theorem and maximal zero-sum free set in $\mathbb{Z} / p \mathbb{Z}$, to appear.

[3] H.Q. Cao and Z.W. Sun, On the number of zero-sum subsequences, Discrete Math. 307 (2007), 1687-1691.

[4] H. Davenport, On the addition of residue classes, J. Lond. Math. Soc. 10 (1935), 30-32.

[5] Z. Füredi and D.J. Kleitman, The minimal number of zero sums, Combinatorics, Paul Erdős is Eighty, J. Bolyai Math. Soc. (1993), 159-172.

[6] P. Erdős, A. Ginzburg and A. Ziv, Theorem in the additive number theory, Bull. Res. Council Israel 10 (1961), 41-43.

[7] W.D. Gao, On a combinatorial problem connected with factorizations, Colloq. Math. 72 (1997), 251-268.

[8] W.D. Gao, On the number of zero-sum subsequences, Discrete Math. 163 (1997), $267-273$.

[9] W.D. Gao, On the number of subsequences with given sum, Discrete Math. 195 (1999), 127-138.

[10] W.D. Gao and A. Geroldinger, Zero-sum problems in finite abelian groups: A survey, Expo. Math. 24 (2006), 337-369.

[11] W.D. Gao and A. Geroldinger, On the number of subsequences with given sum of sequences over finite abelian p-groups, Rocky Mountain J. Math. 37 (2007), 15411550.

[12] W.D. Gao, A. Geroldinger and Q.H. Wang, A quantitative aspect of non-unique factorizations: the Narkiewicz constants, International Journal of Number Theory, to appear.

[13] W.D. Gao and J.T. Peng, On the number of zero-sum subsequences of restricted size, Integers 9 (2009), 537-554.

[14] A. Geroldinger and F. Halter-Koch, Non-unique factorizations, Combinatorial and Analytic Theory, Pure and Applied Mathematics, vol. 278, Chapman \& Hall/CRC, 2006.

[15] A. Geroldinger, Additive group theory and non-unique factorizations, Combinatorial Number Theory and Additive Group Theory, Advanced Courses in Mathematics CRM Barcelona, Birkhäuser, (2009), 1-86.

[16] D.J. Grynkiewicz, On the number of m-term zero-sum subsequences, Acta Arith. 121 (2006), 275-298.

[17] D.J. Grynkiewicz, E. Marchan and O. Ordaz, Representation of finite abelian group elements by subsequence sums, J. Theor. Nombres Bordeaux 21 (2009), 559-587. 
[18] D.R. Guichard, Two theorems on the addition residue classes, Discrete Math. 81 (1990), 11-18.

[19] Y.O. Hamidoune, A note on the addition of residues, Graphs Combin. 6 (1990), $147-152$.

[20] M. Kisin, The number of zero sums modulo $m$ in a sequence of length $n$, Mathematica 41 (1994), 149-163.

[21] W. Narkiewicz, Finite abelian groups and factorization problems, Colloq. Math. 42 (1979), 319-330.

[22] W. Narkiewicz and J. Śliwa, Finite abelian groups and factorization problems II, Colloq. Math. 46 (1982), 115-122.

[23] M.B. Nathanson, Additive Number Theory: Inverse problems and the geometry of sumsets, Vol.165. GTM Springer, New York.

[24] J.E. Olson, A combinatorial problem on finite abelian group II, J. Number Theory 1 (1969), 195-199. 